\title{
The self-avoiding walk spanning a strip
}

\author{
Ben Dyhr * Michael Gilbert ${ }^{\dagger} \quad$ Tom Kennedy ${ }^{\ddagger}$ \\ Gregory F. Lawler ${ }^{\S} \quad$ Shane Passon ^
}

\begin{abstract}
We review the existence of the infinite length self-avoiding walk in the half plane and its relationship to bridges. We prove that this probability measure is also given by the limit as $\beta \rightarrow \beta_{c}$ - of the probability measure on all finite length walks $\omega$ with the probability of $\omega$ proportional to $\beta^{|\omega|}$ where $|\omega|$ is the number of steps in $\omega$. $\left(\beta_{c}\right.$ is the reciprocal of the connective constant.) The self-avoiding walk in a strip $\{z: 0<\operatorname{Im}(z)<y\}$ is defined by considering all self-avoiding walks $\omega$ in the strip which start at the origin and end somewhere on the top boundary with probability proportional to $\beta_{c}^{|\omega|}$ We prove that this probability measure may be obtained by conditioning the SAW in the half plane to have a bridge at height $y$. This observation is the basis for simulations to test conjectures on the distribution of the endpoint of the SAW in a strip and the relationship between the distribution of this strip SAW and $\mathrm{SLE}_{8 / 3}$.
\end{abstract}

\footnotetext{
*Metropolitan State College of Denver

${ }^{\dagger}$ University of Arizona

$\ddagger$ University of Arizona; research supported by NSF grant DMS-0758649.

$\S$ University of Chicago; research supported by NSF grant DMS-0907143.

IUniversity of Arizona
} 


\section{Introduction}

Self-avoiding walks (SAW) on the integer lattice give a simple model of polymers in a dilute solution or more generally random configurations whose only constraint is given by a self-repulsion. In the SAW problem on a lattice, e.g., $\mathbb{Z}^{d}$, one considers nearest neighbor walks on the lattice which visit each lattice site at most once. The basic principle of the standard SAW model is that all walks of a given length (perhaps constrained to have a certain starting point or to stay in a certain subset) are given the same measure. We will consider only $d=2$ in this paper, but the problem is interesting for all $d \geq 2$; see [14] for an overview.

The classic problem for which there are few rigorous results is to understand the asymptotic behavior as $N$ tends to infinity of the uniform probability measure on the set of SAW's of $N$ steps starting at the origin. Among the open questions is a proof that the infinite SAW exists. The latter should be the probability measure on infinite SAW's starting at the origin such that for a finite length SAW $\omega$, the probability of the set of infinite walks that start with $\omega$ is equal to the limit as $N \rightarrow \infty$ of the fraction of SAW's of length $N$ that start with the walk $\omega$. A different question is to find the scaling limit of the infinite SAW which should be very closely related to the scaling limit of the uniform measure. The scaling limit should be obtained by replacing the lattice with unit spacing by a lattice with spacing $\delta$ and letting $\delta$ go to zero.

One can ask a similar question about SAW's restricted to stay in a halfplane. In this case, it can be proved that the infinite half-plane SAW exists. However, it is still open to prove that it has a scaling limit.

It is believed that the SAW is one of the planar models that exhibits conformal invariance in the scaling limit. In [11, see also [10], precise conjectures were made about the kind of scaling limit. We state one version of the conjecture now. Let us write $\mathbb{Z}^{2}=\mathbb{Z}+i \mathbb{Z}$ and let $\mathcal{S}_{y}=\{z \in \mathbb{C}: 0<\operatorname{Im}(z)<y\}$. For each positive integer $y$, consider the set of SAW's starting at 0 , ending at $\{z: \operatorname{Im}(z)=y\}$ and otherwise staying in $\mathcal{S}_{y} \cap \mathbb{Z}^{2}$. To each such walk $\omega$ we assign weight equal to $\beta_{c}^{|\omega|}$. Here $|\omega|$ denotes the number of steps on $\omega$ and $\beta_{c}$ is the inverse of the connective constant defined roughly by saying that the number of SAW's of length $n$ in $\mathbb{Z}^{2}$ starting at the origin grows like $\beta_{c}^{-n}$. It is important to note that although walks of the same length get the same measure, walks of different length are not weighted the same. If $x, y \in \mathbb{Z}$, let $Z_{\mathcal{S}_{y}}(0, x+i y)$ denote the corresponding partition function, that is, the sum of weights of all the walks in $\mathcal{S}_{y}$ that end at $x+i y$ and let 
$Z_{\mathcal{S}_{y}}(0, \mathbb{Z}+i y)=\sum_{x} Z_{\mathcal{S}_{y}}(0, x+i y)$. (It is not obvious that $Z_{\mathcal{S}_{y}}(0, \mathbb{Z}+i y)$ is finite. This was proved in [2], and we give a proof in section 2.4.) Let $\mathbf{Q}_{x}^{y}$, $\mathbf{Q}^{y}$ denote the corresponding probability measures on paths obtained by normalizing by $Z_{\mathcal{S}_{y}}(0, x+i y), Z_{\mathcal{S}_{y}}(0, \mathbb{Z}+i y)$, respectively. Then the conjectures are:

- There exists a scaling exponent $b$ and a scaling function $\rho$ such that

$$
Z_{\mathcal{S}_{y}}(0, x+i y) \sim \rho(x / y) y^{-2 b}, \quad y \rightarrow \infty .
$$

- If $x_{y} \rightarrow \infty$ such that $x_{y} / y \rightarrow x$ then the measures $\mathbf{Q}_{x_{y}}^{y}$ have a scaling limit which is a probability measure $\mu_{x}$ on paths in $\mathcal{S}_{1}$ connecting 0 and $x+i$.

- Similarly, the measures $\mathbf{Q}^{y}$ have a scaling limit which is a probability measure $\mu^{1}$ on paths in $\mathcal{S}_{1}$ connecting 0 and some point on the upper boundary of $\mathcal{S}_{1}$, and which can be written as

$$
\mathbf{Q}^{y} \rightarrow \mu^{1}=c \int_{-\infty}^{\infty} \rho(x) \mu_{x} d x
$$

- The probability measures $\mu_{x}$ are invariant under conformal transformations. For example, if $f: \mathcal{S}_{1} \rightarrow \mathcal{S}_{1}$ is a conformal transformation with $f(0)=0, f(i)=x+i$, then the image of $\mu_{0}$ under $f$ is $\mu_{x}$. (Here probability measures are considered on paths modulo reparametrization.)

More generally, if one has a simply connected domain $D$ and two boundary points $z, w$, one expects to get a probability measure $\mu_{D}(z, w)$ in the scaling limit, and that these measures will be conformally invariant. In general, lattice corrections that persist in the scaling limit will require a more complicated form for the partition function than seen in (11), but we will not worry about this here.

The conjecture (11) suggests that we should have the following scaling rule for the function $\rho$. If $f: \mathcal{S}_{1} \rightarrow \mathcal{S}_{1}$ is a conformal transformation with $f(0)=0, f(i)=x+i$, then

$$
\rho(0)=\left|f^{\prime}(0)\right|^{b}\left|f^{\prime}(i)\right|^{b} \rho(x) .
$$

We give a heuristic argument for (2) in section 3.1. 
In [11], it was shown that if the infinite half-plane SAW has a conformally invariant limit then the limit must be the chordal Schramm-Loewner evolution SLE with exponent $\kappa=8 / 3$. Using this and rigorous results about SLE [12], predictions for critical exponents for the SAW were rederived. In particular, one predicts

$$
b=\frac{5}{8} .
$$

Because these predictions rely on the assumption of the conformally invariant limit, they are not rigorous derivations. This conjecture also led to new predictions about the behavior of SAW, e.g., the probability that a halfplane SAW hits a slit emanating from the real line. These new conjectures were tested numerically in [5, 6], and the simulations were very consistent with the predictions. These simulations give more evidence to believe the assumption of a conformally invariant limit. The work in [5, 6] sampled from the infinite half-plane SAW (more precisely, it sampled from the uniform measure on very long half-plane walks and then restricted the measure to a smaller initial segment of the path), and hence did not give a test of the scaling conjecture for a bounded domain.

The finite scaling rule is difficult to test for two reasons:

- The pivot algorithm, which is the fastest method for producing SAW's, chooses walks from the uniform measure on walks of a fixed number of steps (perhaps restricted to the half-plane). It seems much harder to simulate quickly from the measure where paths are weighted by $\beta_{c}^{|\omega|}$.

- Unless the boundary of our domain consists of straight lines parallel to the coordinate axes, the lattice corrections in the scaling rule (1) are very difficult to understand.

In this paper, we test the conjectures by considering walks in $\mathcal{S}_{y} \cap \mathbb{Z}^{2}$. The limit domain $\mathcal{S}_{1}$ has only horizontal edges, so one does not expect problems in the lattice corrections. Also, as we will show in section 2 , sampling from $\mathbf{Q}^{y}$ is the same as sampling from the infinite half-plane SAW and conditioning on having a "bridge" or "horizontal cut" at $y$. Our work was motivated by [1] who considered bridges of $S L E_{8 / 3}$. Using the prediction $b=5 / 8$ and the scaling rule (2), we will see in section 3.1 that the predicted scaling limit for $\mathrm{Q}^{y}$ is

$$
\mu^{1}=c \int_{-\infty}^{\infty} \rho(x) \mu_{x} d x
$$


where

$$
\rho(x) \propto\left[\cosh \left(\frac{\pi}{2} x\right)\right]^{-5 / 4},
$$

and $\mu_{x}$ is the probability measure associated to chordal $S L E_{8 / 3}$ from 0 to $x+i$ in $\mathcal{S}_{1}$. For the scaling limit of $\mathbf{Q}^{y}$ we consider three main random variables: the distribution of the real part of the endpoint (which should have density $c \rho(x)$ in the limit), the distribution of the rightmost excursion of the path, and the probability that the path passes to the left of a fixed $z \in \mathcal{S}_{1}$. The last probability was first computed for each $\mu_{x}$ by Schramm [15] and one obtains it for $\mu^{1}$ by integrating. We are very pleased to find our simulations give strong numerical evidence for the finite scaling conjectures.

This paper is divided into two parts. The next section is theoretical and establishes mathematically the relationship between the infinite half-plane SAW and the measure on paths weighted by $\beta_{c}^{|\omega|}$. We decided to make this section self-contained except for one difficult estimate of Kesten. In particular, the existence of measures on infinite bridges and half-plane SAW's in [14, 11] are reproved. We include a discussion of half-plane walks started at interior points although we do not do simulations from this measure. In the last section we give a heuristic argument for (2), and then discuss the results of the simulations in detail.

\section{Infinite half-plane SAW}

\subsection{Half-plane SAW starting at the origin}

In this section we review some facts about the infinite self-avoiding half-plane walk. The construction of the measure is due to Madras and Slade [14] who called it the infinite bridge. In [11] it was shown that this measure is in fact the infinite half-plane SAW. Since it is not too difficult, we will give proofs of the results relying only on a difficult estimate due to Kesten.

We write a self-avoiding walk $(\mathrm{SAW})$ in $\mathbb{Z}^{2}=\mathbb{Z} \times i \mathbb{Z}$ as

$$
\omega=\left[\omega_{0}, \ldots, \omega_{n}\right]
$$

We write $|\omega|=n$ for the number of steps of $\omega$. We include the trivial 0-step walks. Let $\mathcal{W}$ denote the set of SAW's in $\mathbb{Z}^{2}$ and $\mathcal{W}_{0}$ the set of $\omega \in \mathcal{W}$ with $\omega_{0}=0$. If $C_{n}=\#\left\{\omega \in \mathcal{W}_{0}:|\omega|=n\right\}$, then the connective constant, which 
we will denote by $1 / \beta_{c}$ is defined by

$$
C_{n} \approx\left(1 / \beta_{c}\right)^{n}, \quad n \rightarrow \infty .
$$

Equivalently, $\beta_{c}$ is the radius of convergence of the power series

$$
\sum_{\omega \in \mathcal{W}_{0}} \beta^{|\omega|}=\sum_{n=0}^{\infty} C_{n} \beta^{n} .
$$

The existence of the connective constant is easily established from the subadditivity relation $C_{n+m} \leq C_{n} C_{m}$ from which one can also conclude that $C_{n} \geq \beta_{c}^{-n}$. The value of the constant is not known; we will only use the simple estimate $0<\beta_{c}<1$.

We will define two different, but related, notions of concatenation of paths. If $\omega^{1}=\left[\omega_{0}^{1}, \ldots, \omega_{m}^{1}\right]$ and $\omega^{2}=\left[\omega_{0}^{2}, \ldots, \omega_{n}^{2}\right]$ are two SAW's with $\omega_{m}^{1}=\omega_{0}^{2}$ we define $\omega^{1} \oplus \omega^{2}$ to be the $(m+n)$-step walk

$$
\omega^{1} \oplus \omega^{2}=\left[\omega_{0}^{1}, \ldots, \omega_{m}^{1}, \omega_{1}^{2}, \ldots, \omega_{n}^{2}\right] .
$$

The concatenation $\omega^{1} \oplus \omega^{2}$ is defined only when the terminal point of $\omega^{1}$ is the same as the initial point of $\omega^{2}$. If $\omega^{2} \in \mathcal{W}_{0}$ we define $\omega^{1} \otimes \omega^{2}$ to be $\omega^{1} \oplus\left(\omega_{m}^{1}+\omega^{2}\right)$, i.e.,

$$
\omega^{1} \otimes \omega^{2}=\left[\omega_{0}^{1}, \ldots, \omega_{m}^{1}, \omega_{m}^{1}+\omega_{1}^{2}, \ldots, \omega_{m}^{1}+\omega_{n}^{2}\right] .
$$

We similarly define $\omega^{1} \oplus \cdots \oplus \omega^{k}, \omega^{1} \otimes \cdots \otimes \omega^{k}$. We write $\omega^{1} \prec \omega$ if we can write $\omega=\omega^{1} \oplus \omega^{2}$ for some $\omega^{2} \in \mathcal{W}$.

\section{Definitions}

- A (upper) half-plane $S A W$ (starting at the origin) is a walk $\omega=\left[\omega_{0}=\right.$ $\left.0, \ldots, \omega_{n}\right] \in \mathcal{W}_{0}$ with $\operatorname{Im}\left[\omega_{j}\right]>0$ for $j>0$. We let $\mathcal{H}$ denote the set of half-plane walks. The height of a walk $\omega \in \mathcal{H}$ is defined to be the maximal value of $\operatorname{Im}\left[\omega_{j}\right], j=0, \ldots, n$. The trivial walk $\omega=[0]$ is the unique $\omega \in \mathcal{H}$ with $h(\omega)=0$. $\mathcal{H}^{*}$ denotes the set of walks $\omega \in \mathcal{H}$ with $h(\omega) \geq 1$, i.e., the nontrivial walks in $\mathcal{H}$.

- A weakly half-plane $S A W$ is a walk $\omega=\left[\omega_{0}=0, \ldots, \omega_{n}\right] \in \mathcal{W}_{0}$ with $\operatorname{Im}\left[\omega_{j}\right] \geq 0$ for $j>0$. We let $\tilde{\mathcal{H}}$ denote the set of weakly half-plane walks. There is a natural bijection between $\tilde{\mathcal{H}}$ and $\mathcal{H}^{*}$ given by

$$
\omega \longleftrightarrow[0, i] \otimes \omega .
$$


- A bridge is a walk $\omega=\left[\omega_{0}=0, \ldots, \omega_{n}\right] \in \mathcal{H}$ with $\operatorname{Im}\left[\omega_{n}\right]=h(\omega)$. The trivial walk $[0]$ is a bridge. We let $\mathcal{B}$ denote the set of bridges and $\mathcal{B}^{*}$ the set of bridges $\omega$ with $h(\omega) \geq 1$, i.e., the nontrivial bridges.

- An irreducible bridge is a bridge $\omega \in \mathcal{B}$ with $h(\omega) \geq 1$ such that $\omega$ cannot be written as

$$
\omega=\omega^{1} \otimes \omega^{2}
$$

with $\omega^{1}, \omega^{2} \in \mathcal{B}^{*}$. We let $\mathcal{I}$ denote the set of irreducible bridges.

- More generally, let $\mathcal{I}_{k}$ denote the set of bridges $\omega$ with $h(\omega) \geq k$ such that $\omega$ cannot be written as

$$
\omega=\omega^{1} \otimes \omega^{2},
$$

with $\omega^{1}, \omega^{2} \in \mathcal{B}^{*}$ and $h\left(\omega^{1}\right) \geq k$. In other words, $\mathcal{I}_{k}$ is the set of bridges that are "irreducible above height $k$ ". Note that $\mathcal{I}=\mathcal{I}_{1}$.

An important observation is that every bridge $\omega \in \mathcal{B}^{*}$ can be written uniquely as

$$
\omega=\omega^{1} \otimes \omega^{2}, \quad \omega^{1} \in \mathcal{I}, \quad \omega^{2} \in \mathcal{B} .
$$

The bridge $\omega$ is irreducible if and only if $\omega^{2}$ is the trivial bridge. More generally, if $h(\omega) \geq k$, then $\omega$ can also be written uniquely as

$$
\omega=\omega^{3} \otimes \omega^{4}, \quad \omega^{3} \in \mathcal{I}_{k}, \quad \omega^{4} \in \mathcal{B} .
$$

We define the generating functions

$$
\begin{gathered}
W(\beta)=\sum_{\omega \in \mathcal{W}_{0}} \beta^{|\omega|}, \quad H(\beta)=\sum_{\omega \in \mathcal{H}} \beta^{|\omega|}, \\
\tilde{H}(\beta)=\sum_{\omega \in \tilde{\mathcal{H}}} \beta^{|\omega|}, \quad B(\beta)=\sum_{\omega \in \mathcal{B}} \beta^{|\omega|}, \\
I_{k}(\beta)=\sum_{\omega \in \mathcal{I}_{k}} \beta^{|\omega|}, \quad I(\beta)=I_{1}(\beta) .
\end{gathered}
$$

The generating functions are increasing in $\beta$ for $\beta>0$ and for such $\beta$, $I_{k}(\beta) \leq B(\beta) \leq H(\beta) \leq W(\beta)$. The bijection between $\tilde{\mathcal{H}}$ and $\mathcal{H}^{*}$ implies

$$
H(\beta)=1+\beta \tilde{H}(\beta) .
$$


Also, eq. (3) gives

$$
B(\beta)=1+I(\beta) B(\beta) .
$$

Recall that $\beta_{c}$ is the radius of convergence of $W(\beta)$. The construction of the measure relies on the following fact due to Kesten [7].

Proposition 2.1. $B\left(\beta_{c}\right)=\infty$ and hence $I\left(\beta_{c}\right)=1$.

Proof. We follow the argument in [14] to prove $B\left(\beta_{c}\right)=\infty$. Since $C_{n} \geq$ $\left(1 / \beta_{c}\right)^{n}, W\left(\beta_{c}\right)=\infty$. By splitting a walk $\omega \in \mathcal{W}_{0}$ at the last point at which the walk achieves its minimal imaginary part, we can see that

$$
W(\beta) \leq \tilde{H}(\beta) H(\beta) .
$$

Using (5), this gives $H\left(\beta_{c}\right)=\infty$. Similarly, each $\omega \in \mathcal{H}$ with $h(\omega) \geq 1$ can be written uniquely as

$$
\omega=\omega^{1} \otimes\left(-\omega^{2}\right) \otimes \omega^{3} \otimes \cdots(-1)^{k-1} \omega^{k},
$$

where $\omega^{1}, \omega^{2}, \ldots, \omega^{k} \in \mathcal{B}$ with $h\left(\omega^{1}\right)>h\left(\omega^{2}\right)>\cdots>h\left(\omega^{k}\right)$. (For example, $\omega^{1}=\left[\omega_{0}, \ldots, \omega_{j}\right]$ where $j$ is the largest index with $\operatorname{Im}\left[\omega_{j}\right]=h(\omega)$.) Let

$$
B_{k}(\beta)=\sum_{\omega \in \mathcal{B}, h(\omega)=k} \beta^{|\omega|} .
$$

Then,

$$
H(\beta) \leq \prod_{k=1}^{\infty}\left[1+B_{k}(\beta)\right] \leq \exp \left\{\sum_{k=0}^{\infty} B_{k}(\beta)\right\}=e^{B(\beta)}, \quad \beta<\beta_{c} .
$$

Hence $B\left(\beta_{c}\right)=\infty$. The second assertion of the proposition follows from (6) which implies that

$$
B(\beta)=\frac{1}{1-I(\beta)} .
$$

This proposition justifies the next definition. In the introduction we used boldface Q's to denote probability measures on SAW's in a strip. $\mathbf{Q}_{x}^{y}$ was a probability measure on SAW's that end at a fixed point on the upper boundary, and $\mathbf{Q}^{y}$ was a probability measure on SAW's that end anywhere on the upper boundary. In the following definition and subsequent definitions we use non-boldface $Q$ 's with subscripts and superscripts to denote probability measures that are defined on SAW's in the upper half-plane. 


\section{Definitions}

- Let $Q$ denote the probability measure on $\mathcal{I}$ defined by

$$
Q(\omega)=\beta_{c}^{|\omega|}, \quad \omega \in \mathcal{I}
$$

- If $j \geq 1$, we consider the product space $\mathcal{I}^{j}$ and define the probability measure $Q^{j}$ by product measure. We also write $Q^{j}$ for the extension to $\mathcal{H}$ with $Q^{j}\left[\mathcal{H} \backslash \mathcal{I}^{j}\right]=0$ and for the corresponding probability measure on $\mathcal{B}$ given by

$$
Q^{j}\left(\omega^{1} \otimes \cdots \otimes \omega^{j}\right)=Q\left(\omega^{1}\right) \cdots Q\left(\omega^{j}\right), \quad \omega^{1}, \ldots, \omega^{j} \in \mathcal{I} .
$$

Here $Q^{j}(\omega)=0$ if $\omega \in \mathcal{B}$ is not of the form $\omega^{1} \otimes \cdots \otimes \omega^{j}, \omega^{1}, \ldots, \omega^{j} \in \mathcal{I}$.

- We define $Q^{\infty}$ on $\mathcal{I}^{\infty}=\mathcal{I} \times \mathcal{I} \times \cdots$, which can be considered as a measure on infinite paths. The infinite half-plane $S A W$ is the measure on infinite self-avoiding paths induced by $Q^{\infty}$.

We have taken this to be the definition. Perhaps we should have defined this to be the infinite bridge as was done by Madras and Slade, but we will show that this definition is equivalent to other definitions that are more naturally called infinite half-plane SAW. There are two natural ways to define an infinite half-plane SAW: either as the limit as $\beta \rightarrow \beta_{c}-$ of walks $\omega \in \mathcal{H}$ weighted by $\beta^{|\omega|}$ or the limit as $n \rightarrow \infty$ of the uniform measure on walks $\omega \in \mathcal{H}$ with $|\omega|=n$. We consider the first of these in this section and discuss the second in Section 2.3 .

Definition If $\beta<\beta_{c}, \mathbf{P}_{\beta}$ denotes the probability measure on $\mathcal{H}$ given by

$$
\mathbf{P}_{\beta}(\omega)=\frac{\beta^{|\omega|}}{H(\beta)}, \quad \omega \in \mathcal{H} .
$$

The next proposition shows that in some sense the limit of $\mathbf{P}_{\beta}$ as $\beta \rightarrow \beta_{c}-$ is the infinite half-plane SAW.

Proposition 2.2. Suppose $\omega^{1}, \ldots, \omega^{j} \in \mathcal{I}$. Let $\mathcal{H}\left(\omega^{1}, \ldots, \omega^{j}\right)$ denote the set of $\omega \in \mathcal{H}$ of the form

$$
\omega=\omega^{1} \otimes \cdots \otimes \omega^{j} \otimes \tilde{\omega}
$$


with $\tilde{\omega} \in \mathcal{H}$. Then if $\beta<\beta_{c}$,

$$
\mathbf{P}_{\beta}\left[\mathcal{H}\left(\omega^{1}, \ldots, \omega^{j}\right)\right]=\beta^{\left|\omega^{1}\right|+\cdots+\left|\omega^{j}\right|} .
$$

In particular,

$$
\lim _{\beta \rightarrow \beta_{c}-} \mathbf{P}_{\beta}\left[\mathcal{H}\left(\omega^{1}, \ldots, \omega^{j}\right)\right]=\beta_{c}^{\left|\omega^{1}\right|+\cdots+\left|\omega^{j}\right|}=Q^{j}\left(\omega^{1} \otimes \cdots \otimes \omega^{j}\right) .
$$

\section{Remarks}

- $\mathbf{P}_{\beta}$ is a probability measure on finite length walks in $\mathbb{H}$, while $Q^{\infty}$ is a measure on infinite length walks in $\mathbb{H}$. The precise sense in which $\mathbf{P}_{\beta}$ converges to $Q^{\infty}$ is the following. In the proposition $\mathcal{H}\left(\omega^{1}, \ldots, \omega^{j}\right)$ denotes the set of finite length walks that start with $\omega^{1} \otimes \cdots \otimes \omega^{j}$. If we let $\mathcal{H}^{\infty}\left(\omega^{1}, \ldots, \omega^{j}\right)$ denote the set of infinite length walks that start with $\omega^{1} \otimes \cdots \otimes \omega^{j}$, then

$$
Q^{\infty}\left[\mathcal{H}^{\infty}\left(\omega^{1}, \ldots, \omega^{j}\right)\right]=\beta_{c}^{\left|\omega^{1}\right|+\cdots+\left|\omega^{j}\right|} .
$$

So the proposition says that

$$
\lim _{\beta \rightarrow \beta_{c}-} \mathbf{P}_{\beta}\left[\mathcal{H}\left(\omega^{1}, \ldots, \omega^{j}\right)\right]=Q^{\infty}\left[\mathcal{H}^{\infty}\left(\omega^{1}, \ldots, \omega^{j}\right)\right]
$$

- Since $Q^{j}$ is a probability measure, a corollary of this proposition is the following. For each $j$ let $A_{j}$ denote the set of walks $\omega \in \mathcal{H}$ that are not of the form

$$
\omega=\omega^{1} \otimes \cdots \otimes \omega^{j} \otimes \tilde{\omega}, \quad \omega^{1}, \ldots, \omega^{j} \in \mathcal{I}, \quad \tilde{\omega} \in \mathcal{H} .
$$

Then

$$
\mathbf{P}_{\beta}\left(A_{j}\right)=1-\sum_{\omega^{1}, \ldots, \omega^{j} \in \mathcal{I}} \beta^{\left|\omega^{1}\right|+\cdots+\left|\omega^{j}\right|}
$$

and hence

$$
\lim _{\beta \rightarrow \beta_{c}-} \mathbf{P}_{\beta}\left(A_{j}\right)=0 .
$$

Proof. Equation (8) follows from

$$
\sum_{\omega \in \mathcal{H}\left(\omega^{1}, \ldots, \omega^{j}\right)} \beta^{|\omega|}=\beta^{\left|\omega^{1}\right|+\cdots+\left|\omega^{j}\right|} H(\beta),
$$

and the rest follows immediately from the definitions. 
Definition If $\omega \in \mathcal{W}$, we say that $\omega$ has a (horizontal) cut at level $k$, if we can write

$$
\omega=\omega^{1} \oplus[j+k i, j+(k+1) i] \oplus \omega^{2},
$$

with $j \in \mathbb{Z}, \omega^{1}, \omega^{2} \in \mathcal{W}$ and $\omega^{1} \subset\{x+i y: y \leq k\}, \omega^{2} \subset\{x+i y: y \geq k+1\}$. Let $\hat{\mathcal{W}}^{k}$ denote the set of walks that have a cut at level $l$ for some $l \geq k$.

Note that if $j>k$ and $\omega$ is of the form (77), then $\omega \in \hat{\mathcal{W}}^{k}$. Since $\mathcal{H} \backslash \hat{\mathcal{W}}^{k} \subset$ $A_{k}$, equation (11) implies for every $k \geq 1$,

$$
\lim _{\beta \rightarrow \beta_{c}-} \mathbf{P}_{\beta}\left(\mathcal{H} \cap \hat{\mathcal{W}}^{k}\right)=1 .
$$

Also, every $\omega \in \mathcal{H} \cap \hat{\mathcal{W}}^{k}$ can be written uniquely as

$$
\omega=\omega^{1} \otimes \omega^{2}, \quad \omega^{1} \in \mathcal{I}_{k}, \quad \omega^{2} \in \mathcal{H}^{*} .
$$

This justifies the next definition.

Definition If $k$ is a positive integer, let $Q_{k}$ denote the probability measure on $\mathcal{I}_{k}$ defined by

$$
Q_{k}(\omega)=\beta_{c}^{|\omega|}, \quad \omega \in \mathcal{I}_{k} .
$$

We also write $Q_{k}$ for the extension to $\mathcal{H}$ with $Q_{k}\left[\mathcal{H} \backslash \mathcal{I}_{k}\right]=0$. It may not be immediately obvious that this is a probability measure. Recall that $\mathcal{I}_{k}$ is the set of bridges which are irreducible above height $k$. In other words, they are of the form $\omega^{1} \otimes \omega^{2} \otimes \cdots \otimes \omega^{j}$ for some $j$ with $\omega^{i} \in \mathcal{I}, h\left(\omega^{1} \otimes \omega^{2} \cdots \otimes \omega^{j-1}\right)<k$, and $h\left(\omega^{1} \otimes \omega^{2} \cdots \otimes \omega^{j}\right) \geq k$. So we can think of the probability measure $Q_{k}$ as follows. We generate a sequence of i.i.d. irreducible bridges distributed according to the probability measure $Q$ and stop when the height of their concatenation is at least $k$.

Note that we have defined two different but similar probability measures $Q^{k}$ and $Q_{k} . Q^{k}$ is a measure on $\mathcal{I}^{k}$, and $Q_{k}$ is a measure on $\mathcal{I}_{k}$. The next proposition is immediate from what we have done so far; it holds for either of these measures.

Proposition 2.3. Suppose $\omega \in \mathcal{H}$ with $h(\omega) \leq j$. Then

$\mathbf{P}_{\beta_{c}}(\{\hat{\omega}: \omega \prec \hat{\omega}\}):=\lim _{\beta \rightarrow \beta_{c}-} \mathbf{P}_{\beta}(\{\hat{\omega}: \omega \prec \hat{\omega}\})=Q^{j}(\{\hat{\omega}: \omega \prec \hat{\omega}\})=Q_{j}(\{\hat{\omega}: \omega \prec \hat{\omega}\})$. 
Proof. We break the set $\{\hat{\omega}: \omega \prec \hat{\omega}\}$ into two disjoint pieces $A$ and $B$. $A$ contains all the $\hat{\omega}$ for which there is an $\bar{\omega} \in \mathcal{I}_{j}$ such that $\omega \prec \bar{\omega} \prec \hat{\omega}$. B contains all the $\hat{\omega}$ for which no such $\bar{\omega}$ exists. Then $A$ is the disjoint union

$$
A=\bigcup_{\bar{\omega} \in \mathcal{I}_{j}: \omega \prec \bar{\omega}} \mathcal{H}(\bar{\omega})
$$

The elements of $B$ are all extensions of $\omega$ which are irreducible above height $j$ and are not bridges. $Q^{j}(\mathcal{H}(\bar{\omega}))=\beta_{c}^{|\bar{\omega}|}=P_{\beta_{c}}(\mathcal{H}(\bar{\omega}))$. $B$ has measure zero under $Q^{j}$ because all the elements have less than $j$ irreducible bridges by construction. From (11) we see $\lim _{\beta \rightarrow \beta_{c}} P_{\beta}(B)=0$. The only elements with positive measure in $Q_{j}$ are those which are bridges and are irreducible above height $j$. Each $\mathcal{H}(\bar{\omega})$ contains only one such SAW, namely $\bar{\omega}$. So $Q_{j}(\mathcal{H}(\bar{\omega}))=Q_{j}(\bar{\omega})=\beta_{c}^{|\bar{\omega}|}$.

\subsection{Half-plane SAW starting at interior point}

We have considered half-plane SAW's that start at a boundary point. We can also consider walks that start at an interior point.

Definition If $z=x+i y \in \mathbb{Z}^{2}$ with $y>0$, let $\mathcal{H}^{z}$ denote the set of walks $\omega=\left[\omega_{0}, \ldots, \omega_{n}\right]$ with $\omega_{0}=z$ and $\operatorname{Im}\left[\omega_{j}\right]>0$ for all $j$. Let

$$
H(\beta, k)=\sum_{\omega \in \mathcal{H}^{k i}} \beta^{|\omega|}=\sum_{\omega \in \mathcal{H}^{x+k i}} \beta^{|\omega|} .
$$

Note that if $\beta \geq 0$, then $H(\beta, k)$ is increasing in $k$; Let $\mathbf{P}_{\beta, z}$ be the probability measure on $\mathcal{H}^{z}$ defined by

$$
\mathbf{P}_{\beta, x+k i}(\omega)=H(\beta, k)^{-1} \beta^{|\omega|} .
$$

In the case $z=i$, there is an obvious bijection between $\mathcal{H}^{i}$ and $\tilde{\mathcal{H}}$. Hence

$$
H(\beta, 1)=\tilde{H}(\beta) \leq \beta^{-1} H(\beta) .
$$

Proposition 2.4. For $\beta<\beta_{c}$ and each $k$,

$$
H(\beta, k) \leq\left(\beta^{-1}+3\right)^{k} H(\beta) .
$$


Remark This estimate is not very sharp but it will be useful. The important fact is that for each $k$, there is a $c_{k}=\left(2 \beta_{c}^{-1}+3\right)^{k}<\infty$ such that for all $\beta_{c} / 2<\beta<\beta_{c}$,

$$
H(\beta, k) \leq c_{k} H(\beta)
$$

Proof. We will proceed by induction; we have already established (13) for $k=1$. Recall that $\beta<\beta_{c}<1$. Suppose $k \geq 2$. We will define an injection

$$
\Phi: \mathcal{H}^{k i} \longrightarrow \bigcup \mathcal{H}^{z}
$$

where the union is over $z \in\{(k-1) i, 1+(k-1) i,-1+(k-1) i,(k-2) i\}$. We partition $\mathcal{H}^{k i}$ into three sets: $W_{1}$, the walks that do not visit $(k-1) i$; $W_{2}$, the walks whose first step is to $(k-1) i$; and $W_{3}$, the walks that visit $(k-1) i$ but not on the first step.

If $\omega \in W_{1}$, let

$$
\Phi(\omega)=[(k-1) i, k i] \oplus \omega .
$$

Then $|\Phi(\omega)|=|\omega|+1$ and the first step of $\Phi(\omega)$ is to $k i$. If $\omega=[k i,(k-$ 1) $i] \oplus \tilde{\omega} \in W_{2}$, let

$$
\Phi(\omega)=\tilde{\omega} .
$$

Then $|\Phi(\omega)|=|\omega|-1$ and $\Phi(\omega)$ does not visit $k i$. In particular, the images $\Phi\left(W_{1}\right)$ and $\Phi\left(W_{2}\right)$ are disjoint, and we can conclude

$$
\sum_{\omega \in W_{1} \cup W_{2}} \beta^{|\omega|} \leq \beta^{-1} H(\beta, k-1) .
$$

If $\omega=\left[\omega_{0}, \ldots, \omega_{n}\right] \in W_{3}$, let $j$ be the vertex with $\omega_{j}=(k-1) i$ and write

$$
\omega=\omega^{1} \oplus \omega^{2},
$$

where $\omega^{1}=\left[\omega_{0}, \ldots, \omega_{j}\right]$. Note that

$$
\omega_{j-1} \in\{(k-2) i, 1+(k-1) i,-1+(k-1) i\} .
$$

Define

$$
\Phi(\omega)=\tilde{\omega}^{1} \oplus \omega^{2} \text { where } \tilde{\omega}^{1}=\left[\omega_{j-1}, \ldots, \omega_{0}, \omega_{j}\right] .
$$

Note that $|\Phi(\omega)|=|\omega|$ and that $\Phi$ maps $W_{3}$ injectively into

$$
\mathcal{H}^{(k-2) i} \cup \mathcal{H}^{1+(k-1) i} \cup \mathcal{H}^{-1+(k-1) i} .
$$


Hence

$$
\sum_{\omega \in W_{3}} \beta^{|\omega|} \leq 2 H(\beta, k-1)+H(\beta, k-2) \leq 3 H(\beta, k-1)
$$

Lemma 2.5. If $k<l$, then

$$
\sum_{\omega \in \mathcal{H}^{k i} \backslash \hat{\mathcal{W}}^{l}} \beta^{|\omega|} \leq\left(\beta^{-1}+3\right)^{k} \sum_{\omega \in \mathcal{H} \backslash \hat{\mathcal{W}}^{l}} \beta^{|\omega|}
$$

In particular,

$$
\lim _{\beta \rightarrow \beta_{c}-} H(\beta, k)^{-1} \sum_{\omega \in \mathcal{H}^{k i} \backslash \hat{\mathcal{W}}^{l}} \beta^{|\omega|}=0 .
$$

Proof. The proof of $(15)$ is the same as the previous proof noting that $\omega \in \hat{\mathcal{W}}^{l}$ if and only if $\Phi(\omega) \in \hat{\mathcal{W}}^{l}$. Since $H(\beta, k)$ increases in $k$,

$$
H(\beta, k)^{-1} \sum_{\omega \in \mathcal{H}^{k i} \backslash \hat{\mathcal{W}}^{l}} \beta^{|\omega|} \leq \frac{\left(\beta^{-1}+3\right)^{k}}{H(\beta)} \sum_{\omega \in \mathcal{H} \backslash \hat{\mathcal{W}}^{l}} \beta^{|\omega|}=\left(\beta^{-1}+3\right)^{k} \mathbf{P}_{\beta}\left(\mathcal{H} \backslash \hat{\mathcal{W}}^{l}\right) .
$$

The final assertion then follows from (12).

Definition If $\omega=\left[x+k i, \omega_{1}, \ldots, \omega_{n}\right] \in \mathcal{H}^{x+k i}$ and $m>k$, we say that $\omega$ is an $m$-irreducible bridge if there is an $l \geq m$ such that $l=\operatorname{Im}\left[\omega_{n}\right]=$ $\max _{1 \leq j \leq n} \operatorname{Im}\left[\omega_{j}\right]$ and $\omega$ has no cuts of level $\geq m$. (Note that an m-irreducible bridge is not necessarily a bridge in the sense described in the previous section.) Let

$$
\tilde{I}(\beta, k ; m)=\sum \beta^{|\omega|},
$$

where the sum is over all $m$-irreducible bridges in $\mathcal{H}^{x+k i}$.

Proposition 2.6. If $m>k$, then

$$
\sum_{\omega \in \mathcal{H}^{k i} \cap \hat{\mathcal{W}}^{m}} \beta^{|\omega|}=\tilde{I}(\beta, k ; m) H(\beta)
$$

In particular,

$$
\tilde{I}\left(\beta_{c}, k ; m\right)=\lim _{\beta \rightarrow \beta_{c^{-}}} \frac{H(\beta, k)}{H(\beta)}<\infty,
$$

so $\tilde{I}\left(\beta_{c}, k, m\right)$ is independent of $m$, and we denote it just by $\tilde{I}\left(\beta_{c}, k\right)$. 
Proof. The first assertion is immediate using the unique decomposition of $\omega \in \mathcal{H}^{k i} \cap \hat{\mathcal{W}}^{m}$ as $\omega=\omega^{1} \otimes \omega^{2}$ where $\omega^{1}$ is an $m$-irreducible bridge and $\omega^{2} \in \mathcal{H}$. From the previous lemma, we see that

$$
H(\beta, k) \sim \sum_{\omega \in \mathcal{H}^{k i} \cap \hat{\mathcal{W}}^{m}} \beta^{|\omega|}, \quad \beta \rightarrow \beta_{c}-.
$$

Using (14), the second assertion follows.

This proposition justifies the following definitions.

\section{Definitions}

- For any $z=x+i k \in \mathbb{Z}^{2}$ with $k>0$ and $m>k$, let $\tilde{Q}_{z, m}$ denote the probability measure on $m$-irreducible bridges in $\hat{\mathcal{H}}^{z}$ given by

$$
\tilde{Q}_{z, m}(\omega)=\frac{\beta_{c}^{|\omega|}}{\tilde{I}\left(\beta_{c}, k\right)} .
$$

- The infinite half-plane SAW starting at $z$ is obtained by choosing

$$
\omega^{1} \otimes \omega^{2} \otimes \cdots,
$$

where $\omega^{1}, \omega^{2}, \ldots$ are independent; $\omega^{1}$ is chosen from $\tilde{Q}_{z, m}$, and $\omega^{2}, \omega^{3}$, ... are chosen from $Q$. (The fact that this definition does not depend on $m$ follows easily from the fact that $\tilde{I}\left(\beta_{c}, k, m\right)$ is independent of $m$.)

Proposition 2.7. Suppose $z=x+i k \in \mathbb{Z}^{2}$ with $k>0$ and $m \geq k$. For each m-irreducible bridge $\omega \in \mathcal{H}^{z}$, let $\mathcal{H}(\omega)$ denote the set of walks $\tilde{\omega} \in \mathcal{H}^{z}$ of the form

$$
\tilde{\omega}=\omega \otimes \omega^{1}, \quad \omega^{1} \in \mathcal{H} .
$$

Then

$$
\lim _{\beta \rightarrow \beta_{c}-} \mathbf{P}_{\beta, z}[\mathcal{H}(\omega)]=\tilde{Q}_{z, m}(\omega)=\frac{\beta_{c}^{|\omega|}}{\tilde{I}\left(\beta_{c}, k\right)} .
$$

Proof. This is proved the same way as proposition 2.3 .

Definition Suppose $V \subset \mathbb{Z}^{2}$ and $z=x+y i \in \mathbb{Z}^{2}$ with $y>0$. Then $\mathcal{H}^{z, V}$ denotes the set of walks $\omega=\left[z, \omega_{1}, \ldots, \omega_{n}\right] \in \mathcal{H}^{z}$ such that $\omega \cap V=\emptyset$. In particular, $\mathcal{H}^{z}=\mathcal{H}^{z, \emptyset}$. 
Proposition 2.8. Suppose $z=x+l i \in \mathbb{Z}^{2}$ with $0<l \leq k, V \subset\{x+i y \in$ $\left.\mathbb{Z}^{2}: y \leq k\right\}$, and $m>k$. Then

$$
\begin{gathered}
\mathbf{P}_{\beta_{c}, z}\left[\mathcal{H}^{z, V}\right]=\sum \tilde{Q}_{z, m}(\omega), \\
\lim _{\beta \rightarrow \beta_{c}-} \sum \beta^{|\omega|}=\mathbf{P}_{\beta_{c}, z}\left[\mathcal{H}^{z, V}\right] \tilde{I}\left(\beta_{c} ; l\right),
\end{gathered}
$$

where the sums are over all $m$-irreducible bridges $\omega \in \mathcal{H}^{z}$ with $\omega \cap V=\emptyset$.

\section{$2.3 \quad$ Limit of counting measure}

Another natural measure on $\mathcal{H}$ is $\mathbf{P}^{n}$, the uniform measure on all walks in $\mathcal{H}$ of length $n$. (We think of $\mathbf{P}^{n}$ as a probability measure on $\mathcal{H}$ by defining $\mathbf{P}(\omega)=0$ if $|\omega| \neq n$.) This is the measure that is easiest to simulate. Here we show that the limit as $n \rightarrow \infty$ of $\mathbf{P}^{n}$ is the infinite half-plane SAW. Let $Y_{n}$ denote the cardinality of $\{\omega \in \mathcal{H}:|\omega|=n\}$. We will use without proof Kesten's result [7]

$$
\lim _{n \rightarrow \infty} \frac{Y_{n}}{Y_{n+2}}=\beta_{c}^{2}
$$

(Kesten proves this for walks in the full plane. His argument works for halfplane walks as well.) Suppose $\omega^{1}, \ldots, \omega^{j} \in \mathcal{I}$ with $m=\left|\omega^{1}\right|+\cdots+\left|\omega^{j}\right|$, and let $\mathcal{H}\left(\omega^{1}, \ldots, \omega^{j}\right)$ be as in Proposition 2.2. Then

$$
\mathbf{P}^{n}\left[\mathcal{H}\left(\omega^{1}, \ldots, \omega^{j}\right)\right]=\frac{Y_{n-m}}{Y_{n}},
$$

where $Y_{k}=0$ if $k<0$.

Proposition 2.9. If $\omega^{1}, \ldots, \omega^{j} \in \mathcal{I}$ with $\left|\omega^{1}\right|+\cdots+\left|\omega^{j}\right|=m$, then

$$
\lim _{n \rightarrow \infty} \mathbf{P}^{n}\left[\mathcal{H}\left(\omega^{1}, \ldots, \omega^{j}\right)\right]=\beta_{c}^{m} .
$$

Remark As before, let $\mathcal{H}^{\infty}\left(\omega^{1}, \ldots, \omega^{j}\right)$ denote the set of infinite length walks that start with $\omega^{1} \otimes \cdots \otimes \omega^{j}$. Then $Q^{\infty}\left[\mathcal{H}^{\infty}\left(\omega^{1}, \ldots, \omega^{j}\right)\right]=\beta_{c}^{m}$. So the proposition says that

$$
\lim _{n \rightarrow \infty} \mathbf{P}^{n}\left[\mathcal{H}\left(\omega^{1}, \ldots, \omega^{j}\right)\right]=Q^{\infty}\left[\mathcal{H}^{\infty}\left(\omega^{1}, \ldots, \omega^{j}\right)\right]
$$

This is the precise sense in which the limit as $n \rightarrow \infty$ of $\mathbf{P}^{n}$ is the infinite half-plane SAW, i.e., $Q^{\infty}$. 
Proof. In [1] it was shown that

$$
\lim _{n \rightarrow \infty} \frac{Y_{n}}{Y_{n+1}}=\beta_{c} .
$$

If we assume this result, then the proof of the proposition is immediate. Instead we give a proof that uses only Kesten's result (16).

Since for fixed $j$,

$$
\sum_{\eta^{1}, \ldots, \eta^{j} \in \mathcal{I}} \beta_{c}^{\left|\eta^{1}\right|+\cdots+\left|\eta^{j}\right|}=1
$$

it suffices to show for every $\omega^{1}, \ldots, \omega^{j} \in \mathcal{I}$

$$
\liminf _{n \rightarrow \infty} \mathbf{P}^{n}\left[\mathcal{H}\left(\omega^{1}, \ldots, \omega^{j}\right)\right] \geq \beta_{c}^{m} .
$$

We will assume that $m$ is even. The case $m$ odd is done the same way. From (16) and (17), we see that

$$
\lim _{n \rightarrow \infty} \mathbf{P}^{2 n}\left[\mathcal{H}\left(\omega^{1}, \ldots, \omega^{j}\right)\right]=\beta_{c}^{m} .
$$

We need to prove also that

$$
\liminf _{n \rightarrow \infty} \mathbf{P}^{2 n+1}\left[\mathcal{H}\left(\omega^{1}, \ldots, \omega^{j}\right)\right] \geq \beta_{c}^{m} .
$$

Suppose $\eta^{1}, \ldots, \eta^{k} \in \mathcal{I}$ with $\left|\eta^{1}\right|, \ldots,\left|\eta^{k-1}\right|$ even and $l=\left|\eta^{1}\right|+\cdots+\left|\eta^{k}\right|$ odd. Then, by the same reasoning

$$
\lim _{n \rightarrow \infty} \mathbf{P}^{2 n+1}\left[\mathcal{H}\left(\omega^{1}, \ldots, \omega^{j}, \eta^{1}, \ldots, \eta^{k}\right)\right]=\beta_{c}^{m+l} .
$$

Note that

$$
\sum^{\dagger} \beta_{c}^{\left|\eta^{1}\right|+\cdots+\left|\eta^{k}\right|}=1
$$

where the sum $\sum^{\dagger}$ is over all $k$ and all $\eta^{1}, \ldots, \eta^{k} \in \mathcal{I}$ such that $\left|\eta^{1}\right|, \ldots,\left|\eta^{k-1}\right|$ are even and $\left|\eta^{k}\right|$ is odd. (Indeed, this is what one gets if one selects $\eta \in \mathcal{I}$ from the distribution $Q$ until one gets a bridge $\eta$ of odd length. Since the probability of getting an $\eta$ of odd length at each step is $\rho>0$ one gets one with probability one.) Similarly,

$$
\mathbf{P}^{2 n+1}\left[\mathcal{H}\left(\omega^{1}, \ldots, \omega^{j}\right)\right] \geq \sum^{\dagger} \mathbf{P}^{2 n+1}\left[\mathcal{H}\left(\omega^{1}, \ldots, \omega^{j}, \eta^{1}, \ldots, \eta^{k}\right)\right] .
$$

where the sum is over $k$ and $\eta^{1}, \ldots, \eta^{k}$ subject to the same constraints as before. Using this, (20) and (21) we get (19). (The reader may observe that the interchange of limits only allows a statement about the liminf rather than a statement about the limit, but we have noted that this suffices.) 
Definition If $A \subset \mathcal{W}$ is any set of SAW's, define

$$
M(A)=\lim _{n \rightarrow \infty} \frac{\#\{\omega \in A:|\omega|=n\}}{Y_{n}},
$$

assuming the limit exists.

Using Proposition 2.6 and a proof as in the previous proposition, we can show that

$$
M\left(\mathcal{H}^{i k}\right)=M\left(\mathcal{H}^{x+i k}\right)=\tilde{I}\left(\beta_{c}, k\right) .
$$

Similarly, as in Proposition 2.8, we have

$$
M\left(\mathcal{H}^{i k, V}\right)=\tilde{I}\left(\beta_{c}, k\right) \mathbf{P}_{\beta_{c}, i k}\left[\mathcal{H}^{i k, V}\right] .
$$

\subsection{SAW in a strip}

In the previous section we considered the self-avoiding walk in the half plane. For every such walk the self-avoidance implies that $\lim _{n \rightarrow \infty}\left|\omega_{n}\right|=\infty$. So the probability measure on the infinite walk is supported on walks between 0 and $\infty$, two boundary points of the upper half plane. In this section we consider the SAW in a strip starting at one boundary point and ending either at a fixed boundary point on the other side of the strip or at any boundary point on the other side of the strip.

We fix a positive integer $y$ and consider the strip

$$
\mathcal{S}_{y}=\{z: 0<\operatorname{Im}(z)<y\}
$$

Fix a point $x+i y$ on the upper boundary of the strip. The SAW from 0 to $x+i y$ in the strip is the probability measure on SAW's that start at 0 , end at $x+i y$ and in between stay in the strip that is defined as follows.

Definition The probability measure $\mathbf{Q}_{x}^{y}$ on the SAW's in the strip $\mathcal{S}_{y}$ from 0 to $x+i y$ is defined by

$$
\mathbf{Q}_{x}^{y}(\omega)=\frac{1}{Z_{\mathcal{S}_{y}}(0, x+i y)} \beta_{c}^{|\omega|}
$$

where $Z_{\mathcal{S}_{y}}(0, x+i y)$ is defined by the requirement that this be a probability measure. 
This definition only makes sense if $Z_{\mathcal{S}_{y}}(0, x+i y)$ is finite. Note that if we take one of the SAW's we are considering and ignore the last step, then we have a bridge with height $y-1$. It need not be irreducible, but it can be decomposed into irreducible bridges. Let $n$ be the number of irreducible bridges. The sum of $\beta_{c}^{|\omega|}$ over the $\omega$ in $Z_{\mathcal{S}_{y}}(0, x+i y)$ that have exactly $n$ irreducible bridges is bounded by 1 by Kesten's relation (Prop. 2.1). Since $n$ is at most $y-1$, it follows that $Z_{\mathcal{S}_{y}}(0, x+i y)$ is finite.

We can also consider SAW's in the strip that start at 0 and end at any point $x+i y$ on the upper boundary. We define a probability measure $\mathbf{Q}^{y}$ on this set of walks in the obvious way.

Definition $\mathbf{Q}^{y}$ is the probability measure on the SAW's in the strip $\mathcal{S}_{y}$ from 0 to some point $z$ with $\operatorname{Im}[z]=y$ given by

$$
\mathbf{Q}^{y}(\omega)=\frac{1}{Z_{\mathcal{S}_{y}}(0, \mathbb{Z}+i y)} \beta_{c}^{|\omega|}
$$

where $Z_{\mathcal{S}_{y}}(0, \mathbb{Z}+i y)$ is defined by the requirement that this be a probability measure.

The same argument that shows $Z_{\mathcal{S}_{y}}(0, x+i y)$ is finite shows $Z_{\mathcal{S}_{y}}(0, \mathbb{Z}+i y)$ is finite. Note that we use a superscript on $\mathbf{Q}$ for probability measures on walks that end anywhere on the upper boundary of the strip and both a subscript and a superscript on $\mathbf{Q}$ for probability measures that end at a prescribed point on the boundary. Obviously, $\mathbf{Q}_{x}^{y}$ can be obtained from $\mathbf{Q}^{y}$ by conditioning on the event that the walk ends at $x+i y$.

Recall that an infinite walk $\omega$ in the half plane has a cut at level $y$ if there is a bridge $\omega_{1}$ with height $y$ and an infinite half plane walk $\omega_{2}$ such that $\omega=\omega_{1} \otimes \omega_{2}$. (Note that this implies that the first bond in $\omega_{2}$ is the only bond in the walk that goes between height $y$ and $y+1$.) The key to our simulations is the following observation.

Proposition 2.10. Let $y$ be a positive integer. Let $Q^{\infty}$ be the probability measure on infinite $S A W^{\prime}$ 's in the half plane from the previous sections. If we condition $Q^{\infty}$ on the event that the walk has a cut at level $y-1$ and only consider the walk up to height $y$, then the resulting probability measure is $\mathbf{Q}^{y}$.

Proof. Let $E_{y-1}$ be the event that $\omega$ has a cut at level $y-1$. Recall that for irreducible bridges $\omega^{1}, \ldots, \omega^{j}, \mathcal{H}^{\infty}\left(\omega^{1}, \ldots, \omega^{j}\right)$ is the set of infinite walks 
that begin with $\omega^{1} \otimes \cdots \otimes \omega^{j}$. We can write $E_{y-1}$ as a disjoint union of these events:

$$
E_{y-1}=\bigcup_{j=1}^{y-1} \bigcup_{\omega^{1}, \ldots, \omega^{j} \in \mathcal{I}: h\left(\omega^{1}\right)+\cdots+h\left(\omega^{j}\right)=y-1} \mathcal{H}^{\infty}\left(\omega^{1}, \ldots, \omega^{j}\right)
$$

By the definition of $Q^{\infty}$,

$$
Q^{\infty}\left[\mathcal{H}^{\infty}\left(\omega^{1}, \ldots, \omega^{j}\right)\right]=\beta_{c}^{\left|\omega^{1}\right|+\cdots+\left|\omega^{j}\right|}
$$

So when we condition on $E_{y-1}$, the probability of $\mathcal{H}^{\infty}\left(\omega^{1}, \ldots, \omega^{j}\right)$ is proportional to $\beta_{c}^{\left|\omega^{1}\right|+\cdots+\left|\omega^{j}\right|}$. If we only consider the walk up to height $y$, then each $\mathcal{H}^{\infty}\left(\omega^{1}, \ldots, \omega^{j}\right)$ corresponds to a single walk, namely, $\omega^{1} \otimes \cdots \otimes \omega^{j}$, concatenated with a vertical bond from height $y-1$ to $y$. So under the conditioning given in the proposition, the probability of a walk in the strip is proportional

to $\beta_{c}^{\left|\omega^{1}\right|+\cdots+\left|\omega^{j}\right|}$. This is what its probability should be under $\mathbf{Q}^{y}$, thus proving the proposition.

\section{Simulations}

\subsection{SLE partition functions}

The pivot algorithm provides a fast Markov chain Monte Carlo algorithm for simulating the SAW in the full plane or the half plane. (For an introduction to this algorithm see [14]. For the fastest implementation of the algorithm to date see [3.) However, the pivot algorithm cannot be used for the SAW in most simply connected domains. The algorithms that do work in these cases are much slower than the pivot algorithm. Proposition 2.2 provides the key to a fast simulation of the SAW in a strip of height $y$. We simulate the SAW in the half plane and condition on the event that the walk has a cut at level $y-1$. The probability of this event is small, but still large enough to allow us to generate large numbers of samples. Proposition 2.10 says that the distribution of the portion of the walk in the strip of height $y$ is $\mathbf{Q}^{y}$.

Of course, the scaling limit of $\mathbf{Q}^{y}$ is not chordal SLE. We could condition further on the event that the walk ends at a particular point $x+i y$ to get $\mathbf{Q}_{x}^{y}$, whose scaling limit should be chordal SLE. However, this would be conditioning on an event with very small probability. Instead we take the following approach. Let $\rho(x)$ be the scaling limit of the density of the unique 
point $x+i y$ on the walk at height $y$ under the measure $\mathbf{Q}^{y}$. If we integrate chordal $\mathrm{SLE}_{8 / 3}$ between 0 and $x+i$ in the strip of height 1 against this density, then we should obtain the scaling limit of $\mathbf{Q}^{y}$. So if we can compute $\rho(x)$, then we can use known results about chordal SLE to make predictions for the scaling limit of $\mathbf{Q}^{y}$. We will derive a conjecture for $\rho(x)$ using SLE partition functions.

For a simply connected domain $D$ and points $z, w$ on its boundary, we define the SLE partition function $H_{D}(z, w)$ by requiring that it be conformally covariant in the following sense. If $\Phi$ is a conformal map on $D$, then

$$
H_{D}(z, w)=\left|\Phi^{\prime}(z) \Phi^{\prime}(w)\right|^{5 / 8} H_{\Phi(D)}(\Phi(z), \Phi(w))
$$

This defines $H_{D}(z, w)$ up to specifying its value for one particular choice of $D, z, w$. We follow the usual convention of taking $H_{\mathbb{H}}(0,1)=1$. This partition function is believed to be related to the total mass of the scaling limit of SAW's in $D$ from $z$ to $w$. This statement must be interpreted with caution as there are lattice effects that persist in the scaling limit. We will motivate this conjecture by arguing that it is true in two special cases.

In the first special case, $D$ will be $\mathbb{H}$ and $\Phi$ will just be a dilation. Let

$$
Z_{\mathbb{H}}(0, n)=\sum_{\omega: 0 \rightarrow n, \omega \subset \mathbb{H}} \beta_{c}^{|\omega|}
$$

The sum is over all self-avoiding walks that start at 0 , end at $n$ and stay in the upper half plane except for their endpoints. We assume that there is an exponent $\rho$ such that the limit

$$
c=\lim _{n \rightarrow \infty} Z_{\mathbb{H}}(0, n) n^{\rho}
$$

exists. There is not a proof that $Z_{\mathbb{H}}(0, n)$ is finite. Now let $x>0$. Let $[n x]$ denote the integer closest to $x$. We have

$$
\lim _{n \rightarrow \infty} Z_{\mathbb{H}}(0,[n x]) n^{\rho}=\lim _{n \rightarrow \infty} Z_{\mathbb{H}}(0,[n x])[n x]^{\rho} \frac{n^{\rho}}{[n x]^{\rho}}=\frac{c}{x^{\rho}}
$$

This shows that

$$
\lim _{n \rightarrow \infty} \frac{Z_{\mathbb{H}}(0,[n x])}{Z_{\mathbb{H}}(0, n)}=\frac{1}{x^{\rho}}
$$

If we use (23) with $\Phi$ equal to a dilation, then we have

$$
\frac{H_{\mathbb{H}}(0, x)}{H_{\mathbb{H}}(0,1)}=H_{\mathbb{H}}(0, x)=\frac{1}{x^{5 / 4}} .
$$


So if (24) is true with $\rho=5 / 4$, then $H_{\mathbb{H}}(0, x)$ does indeed give the relative weight of the scaling limit of the SAW from 0 to $x$ in $\mathbb{H}$.

For the argument for the second special case of (23) we use the following result of Lawler, Schramm and Werner [12].

Theorem 3.1. Suppose $A \subset \overline{\mathbb{H}}$ is compact and $\mathbb{H} \backslash A$ is simply connected with $0 \notin A$. Let $\mathbf{P}_{\mathbb{H}, 0, \infty}$ denote the probability measure on simple curves in $\mathbb{H}$ from 0 to $\infty$ given by chordal $S L E_{8 / 3}$. If $\Phi_{A}: \mathbb{H} \backslash A \rightarrow \mathbb{H}$ denotes the unique conformal map that fixes 0 and $\infty$ and has $\Phi_{A}^{\prime}(\infty)=1$, then

$$
\mathbf{P}_{\mathbb{H}, 0, \infty}[\gamma \cap A=\emptyset]=\Phi_{A}^{\prime}(0)^{5 / 8}
$$

Let $D^{\prime}$ be a simply connected domain and $z, w$ two points on its boundary. Let $D$ be a simply connected domain which is a subset of $D^{\prime}$ and such that $z, w$ also belong to the boundary of $D$. Let $\Phi$ be a conformal map of $D$ to $D^{\prime}$ which fixes $z$ and $w$. Then the definition of the SLE partition function says

$$
H_{D}(z, w)=\left|\Phi^{\prime}(z) \Phi^{\prime}(w)\right|^{5 / 8} H_{D^{\prime}}(z, w)
$$

Now consider the total mass of the SAW in $D$ from $z$ to $w$ divided by the total mass of the SAW in $D^{\prime}$ from $z$ to $w$. This ratio gives the probability that a SAW in $D^{\prime}$ from $z$ to $w$ remains in the smaller domain $D$. This should be given by the probability that a chordal $\mathrm{SLE}_{8 / 3}$ in $D^{\prime}$ from $z$ to $w$ remains in $D$, i.e., by $\mathbf{P}_{D^{\prime}, z, w}\left(\gamma \cap\left(D^{\prime} \backslash D\right)=\emptyset\right)$, where $\mathbf{P}_{D^{\prime}, z, w}$ denotes this chordal SLE measure. We can compute this probability using Theorem 3.1. We will show that it is given by $\left|\Phi^{\prime}(z) \Phi^{\prime}(w)\right|^{5 / 8}$, i.e., by the ratio of the SLE partition functions $H_{D}(z, w) / H_{D^{\prime}}(z, w)$.

Let $\psi(z)$ be a conformal map of $D^{\prime}$ to $\mathbb{H}$ with $\psi(z)=0$ and $\psi(w)=\infty$. By the conformal invariance of SLE,

$$
\mathbf{P}_{D^{\prime}, z, w}\left(\gamma \cap\left(D^{\prime} \backslash D\right)=\emptyset\right)=\mathbf{P}_{\mathbb{H}, 0, \infty}\left(\gamma \cap \psi\left(D^{\prime} \backslash D\right)=\emptyset\right) .
$$

Let $\phi=\psi \circ \Phi \circ \psi^{-1}$. Then $\phi$ maps $\mathbb{H} \backslash \psi\left(D^{\prime} \backslash D\right)$ to $\mathbb{H}$ and fixes 0 and $\infty$. So it satisfies the hypotheses of Theorem 3.1 except that its derivative at $\infty$ need not be 1 . An easy computation shows $\phi(z) \sim z / \Phi^{\prime}(w)$ as $z \rightarrow \infty$. So $z \mapsto \Phi^{\prime}(w) \phi(z)$ satisfies the hypotheses. The chain rule shows $\phi^{\prime}(0)=\Phi^{\prime}(z)$, SO

$$
\mathbf{P}_{\mathbb{H}, 0, \infty}\left(\gamma \cap \psi\left(D^{\prime} \backslash D\right)=\emptyset\right)=\left|\Phi^{\prime}(z) \Phi^{\prime}(w)\right|^{5 / 8}
$$

Thus we have derived (23) for the special case that $\Phi(D)$ is a superset of $D$ with $z, w$ on its boundary too, and $\Phi$ fixes $z$ and $w$. 


\subsection{Boundary density for the strip}

Our simulations are all for the SAW in a strip. We use $\mathcal{S}$ to denote the strip of height 1, i.e., $\{z \in \mathbb{H}: 0<\operatorname{Im}(z)<1\}$. We use SLE partition functions to compute the conjecture for the boundary density for the scaling limit of the SAW in $\mathcal{S}$ starting at 0 and ending somewhere on the upper boundary of the strip. So we need to compute $H_{\mathcal{S}}(0, x+i)$. Let $f(z)=e^{\pi z}-1$. This sends the strip to the upper half plane, sending 0 to 0 and $x+i$ to $-e^{\pi x}-1$. We have $f^{\prime}(0)=\pi, f^{\prime}(x+i)=-\pi e^{\pi x}$. So using (23) and the special case (25), we have

$$
\begin{aligned}
H_{\mathcal{S}}(0, x+i) & =\left(\pi^{2} e^{\pi x}\right)^{5 / 8} H_{\mathbb{H}}\left(0,-e^{\pi x}-1\right) \\
& =\left[\frac{\pi^{2} e^{\pi x}}{\left(e^{\pi x}+1\right)^{2}}\right]^{5 / 8}=\left[\frac{\pi^{2}}{4 \cosh ^{2}(\pi x / 2)}\right]^{5 / 8}
\end{aligned}
$$

Thus our conjecture for the density for the SAW in the strip from 0 to the point $x+i$ on the upper boundary is

$$
\rho(x)=c\left[\cosh \left(\frac{\pi}{2} x\right)\right]^{-5 / 4}, \quad-\infty<x<\infty
$$

where the constant $c$ normalizes the density.

Our simulations generated SAW's in the half plane with $N=10,000$ steps. We condition on the event that the walk has a cut at a fixed level $h$ which we take to be $h=0.2 N^{3 / 4}=200$. In practice this means that we run the pivot algorithm to generate a sequence of SAW's. These walks are highly correlated, so we only look at the walk every 100,000 steps of the Markov chain to see if the walk has a cut at the prescribed level. If it does, we use it to compute a sample of the random variables we are studying. We generated a total of 496,000 samples. For the particular $N$ and $h$ that we used, approximately $27 \%$ of the SAW's have a cut at the prescribed height. We expect this fraction to decrease to zero as $h$ goes to infinity.

Figure 1 shows our test of conjecture (30) for the boundary density. We plot the cumulative distribution for this density for a simulation of the SAW and for the conjectured density. The two curves are indistinguishable on the scale of the figure. The left inset shows a blow-up of a section of the two curves to show the size of the difference. The right inset is a plot of the difference of the theoretical and empirical distribution. This difference is typically on the order of $1 / 10$ of a percent. The very jagged nature of this curve is a reflection of the effect of the nonzero lattice spacing. On a small scale the simulation is actually simulating a discrete random variable. 


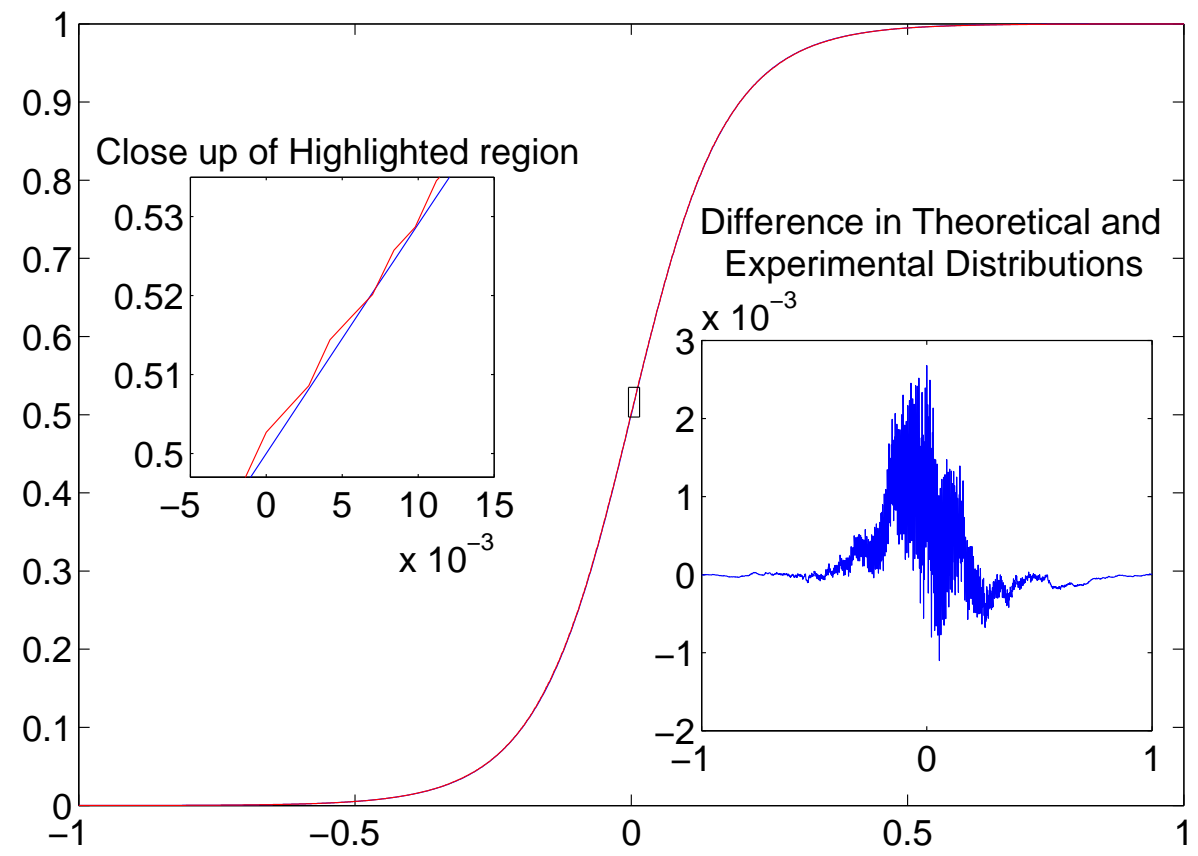

Figure 1: Comparison of simulations of boundary density for the SAW in a strip and the conjectured density using SLE partition functions.

\subsection{Passing left of a point}

Now that we have found $\rho(x)$, we can use it together with results for chordal $\mathrm{SLE}_{8 / 3}$ to make predictions for the scaling limit of $\mathbf{Q}^{y}$. The probability of certain random events depending on the geometry of the $\mathrm{SLE}_{8 / 3}$ curve can be explicitly computed. We have already seen one such formula in Theorem 
3.1 .

The second formula for chordal SLE that we will use is Schramm's leftpassing probability of a point $z \in \mathbb{H}$ with respect to the $\mathrm{SLE}_{\kappa}$ generating curve [15]. (Schramm called this a 'left crossing probability,' but we use slightly different terminology here.) Schramm's formula applies to any $\kappa \in(0,8)$. The definition of left-passing is given in terms of winding numbers. For $\kappa=8 / 3$, the generating curve is a simple curve; an equivalent definition of left-passing is

$$
\mathbf{P}_{\mathbb{H}, 0, \infty}[\gamma \text { passes left of } z]=\mathbf{P}_{\mathbb{H}, 0, \infty}\left[z \in H_{\infty}^{+}\right]
$$

where $H_{\infty}^{+}$is defined to be the connected component of $\overline{\mathbb{H}} \backslash \gamma[0, \infty)$ that contains $\mathbb{R}^{+}:=\{x \in \mathbb{R}: x>0\}$. Since $\gamma$ is simple and $\gamma(t) \rightarrow \infty$ w.p.1, $\overline{\mathbb{H}} \backslash \gamma[0, \infty)$ has exactly two connected components. For $\kappa=8 / 3$, the formula reduces to

$$
\mathbf{P}_{\mathbb{H}, 0, \infty}[\gamma \text { passes left of } z]=\frac{1}{2}[1+\cos (\arg (z))] \text {. }
$$

For the SAW in the half plane, simulations were compared with the leftcrossing formula and with several applications of Theorem 3.1 in [5, 6]. Excellent agreement was found. We emphasize that these past Monte Carlo tests of SLE predictions for the SAW have all been for the SAW in the half plane or in a slit full plane. The simulations in this paper are the first tests of SLE predictions for the SAW in a strip. More significantly, they are the first tests of the SLE partition function prediction (30).

Recall that $\mathcal{S}=\{z \in \mathbb{H}: 0<\operatorname{Im}(z)<1\}$. The map

$$
\Psi_{\xi}(z)=\frac{e^{\pi z}-1}{e^{\pi z}+e^{\pi \xi}}
$$

maps $\mathcal{S}$ to $\mathbb{H}$ with $\xi+i \mapsto \infty$ and $0 \mapsto 0$. We use this map to transform probabilities involving chordal SLE in the strip into chordal SLE in the half plane.

If $z$ is a point in the interior of the $\operatorname{strip} \mathcal{S}$, then

$$
\begin{aligned}
\mathbf{P}_{\mathcal{S}, 0, x+i}(\gamma \text { passes left of } z) & =\mathbf{P}_{\mathbb{H}, 0, \infty}\left(\gamma \text { passes left of } \Psi_{x}(z)\right) \\
& =\frac{1}{2}\left[1+\cos \left(\arg \left(\Psi_{x}(z)\right)\right)\right] .
\end{aligned}
$$

So we expect that

$$
\lim _{y \rightarrow \infty} \mathbf{Q}^{y}\left(\frac{\omega}{y} \text { passes left of } z\right)=\int_{-\infty}^{\infty} \frac{1}{2}\left[1+\cos \left(\arg \left(\Psi_{x}(z)\right)\right)\right] \rho(x) d x
$$


In our simulations we sample the event that the walk passes left of $z$ for a grid of values of $z$. The grid is 400 points wide horizontally and 100 points wide vertically. We then use the probabilities of these events to compute a contour plot for the probability of the curve passing left as a function of $z$. Figure 2 shows the comparison of the theoretical probabilities and the simulations. Contour plots were generated for the contours for probabilities $0.1,0.2,0.3, \ldots, 0.8,0.9$. The solid lines are the theoretical contours and the circle are points on the empirical contours at $y$ values of $0.05,0.10,0.15, \ldots, 0.95$.



Figure 2: Comparison of simulations of the probability of passing left for the SAW in a strip and the conjectured probability function using SLE. The plot is a contour plot in the strip which shows the curves where the probability is $0.1,0.2, \ldots, 0.9$. 


\subsection{Right-most excursion}

Our next test considers the right-most point on the SAW in the strip, i.e., the random variable

$$
\max _{0 \leq j \leq|\omega|} \operatorname{Re} \omega_{j}
$$

We conjecture that in the scaling limit its distribution is given by

$$
\lim _{y \rightarrow \infty} \mathbf{Q}^{y}\left(\max _{j} \frac{\operatorname{Re} \omega_{j}}{y} \leq x\right)=\int_{-\infty}^{\infty} \mathbf{P}_{\mathcal{S}, 0, \xi+i}\left(\max _{t} \operatorname{Re} \gamma(t) \leq x\right) \rho(\xi) d \xi
$$

where $\mathbf{P}_{\mathcal{S}, 0, \xi+i}$ denotes the probability measure for chordal $\mathrm{SLE}_{8 / 3}$ in the strip $\mathcal{S}$ from 0 to $x+i$.

To compute $\mathbf{P}_{\mathcal{S}, 0, \xi+i}\left(\max _{t} \operatorname{Re} \gamma(t) \leq x\right)$, we first note that this probability is zero if $\xi>x$. For $\xi<x$, we observe that the random variable is $<x$ if and only if the curve does not hit the portion of the strip given by $\operatorname{Re} z \geq x$. So for $\xi<x$ we have

$$
\begin{aligned}
\mathbf{P}_{\mathcal{S}, 0, \xi+i}\left(\max _{t} \operatorname{Re} \gamma(t)<x\right) & =\mathbf{P}_{\mathcal{S}, 0, \xi+i}(\gamma \cap\{z \in \mathcal{S}: \operatorname{Re} z \geq x\}=\emptyset) \\
& =\mathbf{P}_{\mathbb{H}, 0, \infty}\left(\gamma \cap \Psi_{\xi}(\{z \in \mathcal{S}: \operatorname{Re} z \geq x\})=\emptyset\right) \\
& =\mathbf{P}_{\mathbb{H}, 0, \infty}(\gamma \cap\{z \in \mathbb{H}:|z-a(x ; \xi)| \leq c(x ; \xi)\}=\emptyset)
\end{aligned}
$$

where $a(x ; \xi)$ and $c(x ; \xi)$ are given by,

$$
\begin{aligned}
& a(x ; \xi)=\frac{1}{2}\left(\frac{\exp \pi x-1}{\exp \pi x+\exp \pi \xi}+\frac{\exp \pi x+1}{\exp \pi x-\exp \pi \xi}\right) \text { and } \\
& c(x ; \xi)=\frac{1}{2}\left|\frac{\exp \pi x-1}{\exp \pi x+\exp \pi \xi}-\frac{\exp \pi x+1}{\exp \pi x-\exp \pi \xi}\right| .
\end{aligned}
$$

We can now appeal to equation (26). The map $\Phi_{A}$ in (26) is given by

$$
\Phi_{A}(z)=\frac{(c-a)^{2}}{a}\left(\frac{\left(\frac{c-(z-a)}{c+(z-a)}\right)^{2}-\left(\frac{c+a}{c-a}\right)^{2}}{\left(\frac{c-(z-a)}{c+(z-a)}\right)^{2}-1}\right) .
$$

where $a=a(x ; \xi), c=c(x ; \xi)$.

Evaluating the derivative at $z=0$ gives

$$
\lim _{y \rightarrow \infty} \mathbf{Q}^{y}\left(\max _{j} \frac{\operatorname{Re} \omega_{j}}{y} \leq x\right)=\int_{-\infty}^{x}\left[1-\left(\frac{c(x ; \xi)}{a(x ; \xi)}\right)^{2}\right]^{5 / 8} \rho(\xi) d \xi .
$$


Figure 3 shows the comparison of this prediction and our simulations. The two curves are indistinguishable on the scale of the main figure. The left inset shows a blow up of a small section where they deviate the most. (The dashed line is the simulation distribution.) The right inset shows a plot of the difference of the two curves. This difference is less than $1 / 10$ of a percent.

\section{Conclusions}

We have reviewed the construction of the probability measure on infinite SAW's in the half plane as an i.i.d. sequence of irreducible bridges and the proof that this measure is the weak limit as $N \rightarrow \infty$ of the uniform probability measure on SAW's with $N$ steps. We have shown that the measure on infinite length SAW's is the weak limit as $\beta \rightarrow \beta_{c}$ - of the probability measure on all finite length SAW's in which the probability of a SAW $\omega$ is proportional to $\beta^{|\omega|}$.

We have considered the SAW in a strip of height $y$ which starts at the origin and ends anywhere on the upper boundary. The probability measure in which the probability of $\omega$ is proportional to $\beta_{c}^{|\omega|}$ can also be obtained by taking the SAW in the half plane and conditioning on the event that $y$ is a bridge height for the SAW.

Using this relationship we have carried out simulations of the SAW in the strip and found good agreement with the conjecture of Lawler, Schramm and Werner for the density of the endpoint of the SAW along the upper boundary. This is the first test of their prediction for such boundary densities. Our simulations have also tested the conjecture that the scaling limit of the SAW is $\mathrm{SLE}_{8 / 3}$ for the strip and found good agreement.

Our simulations have provided the first test of the conjectured conformal covariance of the boundary density, but the strip is a rather special test case since the boundary is always parallel to a lattice direction. It is expected that even in the scaling limit there will be lattice effects that must be taken into account for other domains. This merits further study. 


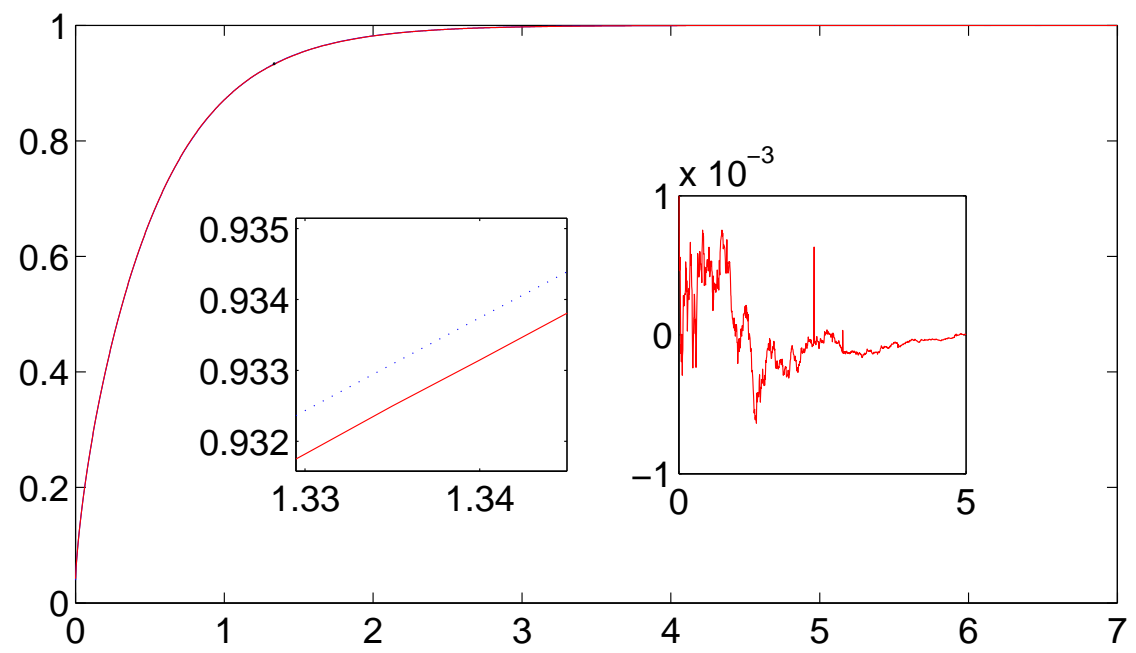

Figure 3: Comparison of simulation of right most excursion of the SAW in a strip and the conjectured distribution function using SLE. The simulation distribution is the dashed line. 


\section{References}

[1] T. Alberts, H. Duminil-Copin, Bridge decomposition of restriction measures, J. Stat. Phys. 140, 467-493 (2010). Archived as arXiv:0909.0203v1 [math.PR].

[2] J. T. Chayes, L. Chayes, Ornstein-Zernike behavior for self-avoiding walks at all noncritical temperatures, Commun. Math. Phys. 105, 221238 (1986).

[3] N. Clisby, Efficient implementation of the pivot algorithm for self-avoiding walks, J. Stat. Phys. 140, 349-392 (2010). Archived as arXiv:1005.1444v1 [cond-mat.stat-mech].

[4] T. Kennedy, The length of an SLE - Monte Carlo studies, J. Stat. Phys. 128, 1263-1277 (2007). Archived as arXiv:math/0612609v2 [math.PR].

[5] T. Kennedy, Monte Carlo tests of SLE predictions for 2D selfavoiding walks, Phys. Rev. Lett. 88, 130601 (2002). Archived as arXiv:math/0112246v1 [math.PR].

[6] T. Kennedy, Conformal invariance and stochastic Loewner evolution predictions for the 2D self-avoiding walk - Monte Carlo tests, J. Stat. Phys. 114, 51-78 (2004). Archived as arXiv:math/0207231v2 [math.PR].

[7] H. Kesten, On the number of self-avoiding walks, J. Math. Phys 4, 960969 (1963).

[8] H. Kesten, On the number of self-avoiding walks II, J. Math. Phys 5, 1128-1137 (1964).

[9] G. Lawler, Conformally Invariant Processes in the Plane. American Mathematical Society (2005).

[10] G. Lawler, Schramm-Loewner evolution, in Statistical Mechanics, S. Sheffield and T. Spencer, ed., IAS/Park City Mathematical Series, AMS, 231-295 (2009). Archived as arXiv:0712.3256v1 [math.PR].

[11] G. Lawler, O. Schramm, and W. Werner, On the scaling limit of planar self-avoiding walk, Fractal Geometry and Applications: a Jubilee of Benoit Mandelbrot, Part 2, 339-364, Proc. Sympos. Pure Math. 72, Amer. Math. Soc., Providence, RI, 2004. Archived as arXiv:math/0204277v2 [math.PR] 
[12] G. Lawler, O. Schramm, W.Werner, Conformal restriction: the chordal case, J. Amer. Math. Soc. 16, 917-955 (2003). Archived as arXiv:math/0209343v2 [math.PR].

[13] G. Lawler, S. Sheffield, The natural parametrization for the SchrammLoewner evolution, preprint (2009). Archived as arXiv:0906.3804v1 [math.PR].

[14] N. Madras and G. Slade, The Self-Avoiding Walk. Birkhäuser (1996).

[15] O. Schramm, A percolation formula, Electron. Comm. Probab. 6, 115120 (2001). Archived as arXiv:math/0107096v2 [math.PR]. 